\title{
Supporting Information: Asymmetric Deposition of Platinum Atoms on Gold Nanorods Reduced the Plasmon Field Distortion Induced by the Substrate
}

Lourdes Bazán-Díaz, ${ }^{\dagger}$ Rubén Mendoza-Cruz, ${ }^{\dagger}$ Chih-Kai Liao, ${ }^{\dagger}$ Mahmoud A. Mahmoud ${ }^{\dagger, \pm,+t^{*}}$

Chemical Engineering, Department of Biomedical Engineering, ${ }^{\dagger}$ Department of Chemistry, \pm Department of Physics and Astronomy, the University of Texas at San Antonio, One UTSA Circle, San Antonio, TX 78249

*Mahmoud.abdelwahed@UTSA.edu 
Gold nanorods gold nanorods (AuNRs) were prepared by the seed-mediated technique as follows: Synthesis of $A \boldsymbol{u}$ seeds: In a $30 \mathrm{~mL}$ vial, $5 \mathrm{~mL}$ of a $0.2 \mathrm{M}$ aqueous solution of cetyltrimethylammonium bromide (CTAB) was mixed with $2.5 \mathrm{~mL}$ of a $1.0 \mathrm{mM}$ aqueous solution of $\mathrm{HAuCl}_{4} .0 .6 \mathrm{~mL}$ of a $10 \mathrm{mM}$ ice-cold sodium borohydride solution was added to the stirred CTAB-gold ion solution. The resulting solution was stirred for $5 \mathrm{~min}$.

Growth of the seeds to AuNRs: In $500 \mathrm{~mL}$ flask $200 \mathrm{~mL}$ of $0.2 \mathrm{M}$ aqueous solution of CTAB were mixed with $200 \mathrm{~mL}$ of a $1.0 \mathrm{mM} \mathrm{HAuCl}_{4}$ aqueous solution. $9 \mathrm{~mL}$ of a $4.0 \mathrm{mM} \mathrm{AgNO}_{3}$ solution was added to the resulting solution followed by $2.8 \mathrm{~mL}$ of $78.8 \mathrm{mM}$ ascorbic acid. Finally, $320 \mu \mathrm{L}$ seed solution was added and the solution left to react overnight. The synthesized colloidal AuNRs were centrifuged at $8000 \mathrm{rpm}$ for $10 \mathrm{~min}$ in $50 \mathrm{~mL}$ tubes. The precipitate was dispersed in deionized water and centrifuged one more time at $5000 \mathrm{rpm}$ for $5 \mathrm{~min}$. The precipitated AuNRs were redispersed in $50 \mathrm{~mL}$ of deionized water.

Functionalization AuNRs with PEG: $0.3 \mathrm{~mL}$ of $1 \mathrm{mM}$ thiolated poly(ethylene glycol) (PEG; Laysan Bio, $\mathrm{Mn}=30,000)$ dispersed in water was added to the AuNRs solution and stirred overnight at $800 \mathrm{rpm}$ to bound the PEG to the surface of AuNRs via the thiol group. The functionalized AuNRs were centrifugated twice at $6000 \mathrm{rpm}$ for $10 \mathrm{~min}$ to remove the excess of PEG, and the precipitated was dispersed in methanol. Finally, the AuNRs were precipitated at $5000 \mathrm{rpm}$ for $15 \mathrm{~min}$ and dispersed in a mixture of $4 \mathrm{~mL}$ of chloroform and $2 \mathrm{~mL}$ of methanol.

Fabrication of AuNR 2-D arrays: 2-D arrays of AuNRs were fabricated by spraying AuNRs in chloroform-ethanol solution over the water sublayer of a Langmuir Blodgett trough by microsyringe. The 2-D arrays were then transferred to the surface of a glass substrate via vertical dipping with a speed of $2 \mathrm{~mm} / \mathrm{min}$. The, the arrays were allowed to dry for $10 \mathrm{~min}$ in air before being left in a desiccator for $2 \mathrm{~h}$. 


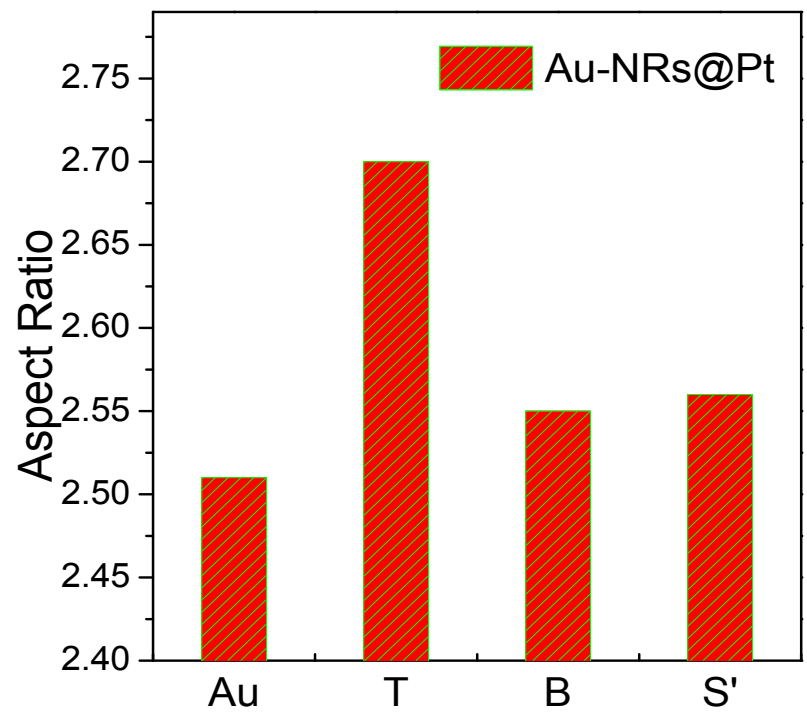

Figure S1. Aspect ratio evolution of Au-NRs covered with Pt at different deposition time: $\mathrm{T}=10$ min, $B=30$ min, and $S=60$ min.
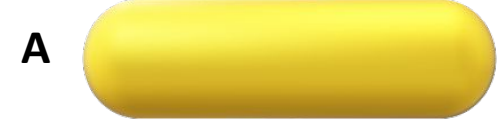

C
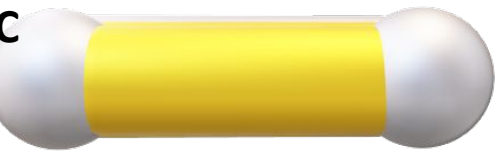

B

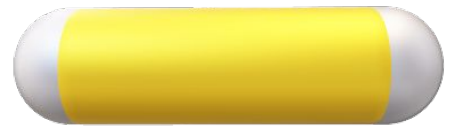

D

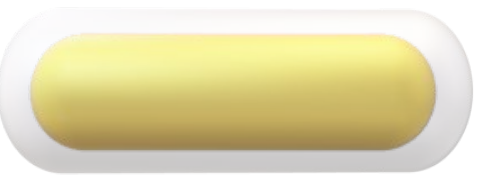

Figure S2. Geometrical simplified models of the coverage types of Pt over Au-NRs. (A) Pure Au-NRs, (b) Tip-enhanced, (C) Bone type, and (D) Shell. 

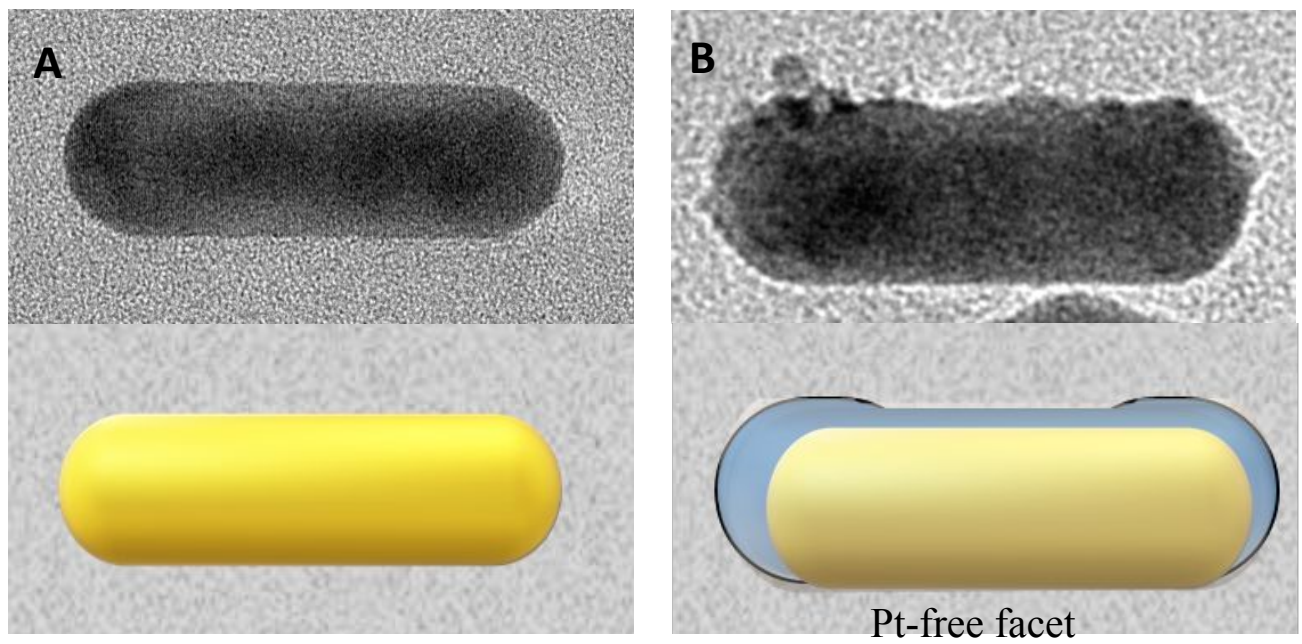

Figure S3. Magnified TEM image and geometrical model of the (A) pure Au-NRs, and (B) Ptcovered Au-NRs where a platinum-free facet is formed by the substrate.
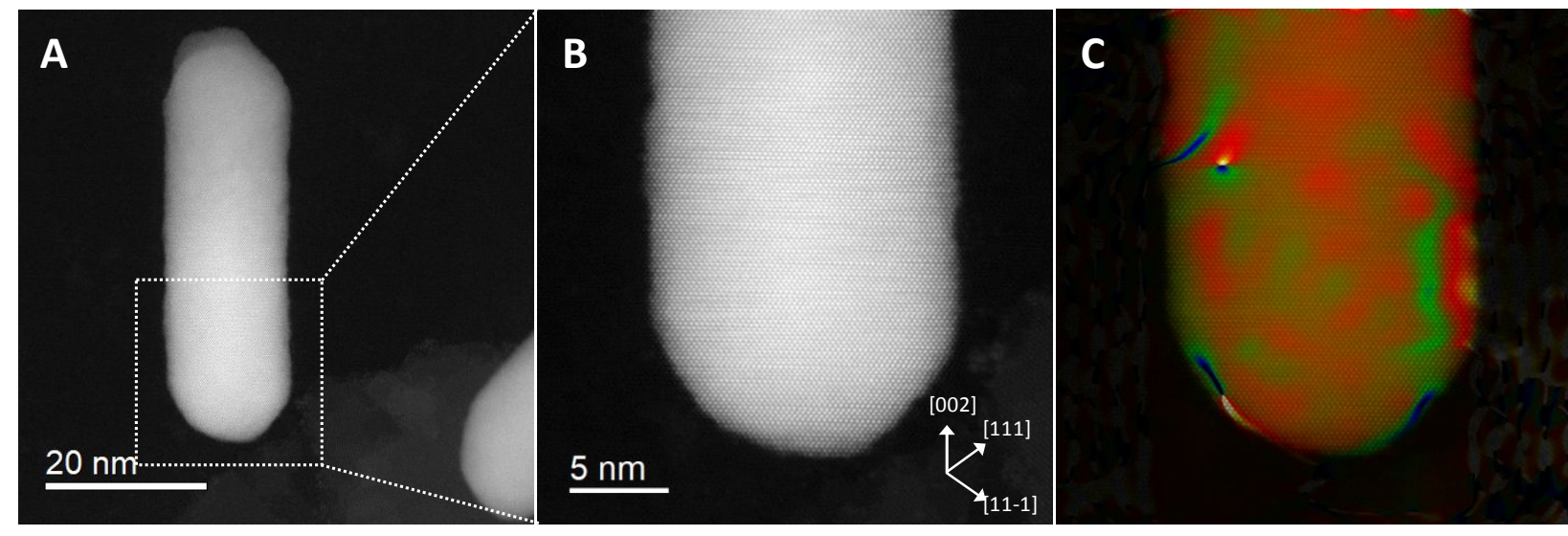

Figure S4. (A,B) HAADF-STEM image of the bottom tip of the Pt@AuNR-T shown in Figure 3, and (C) GPA of the tip of a single nanorod showing a large lattice strain suggesting the deposition of Pt. 

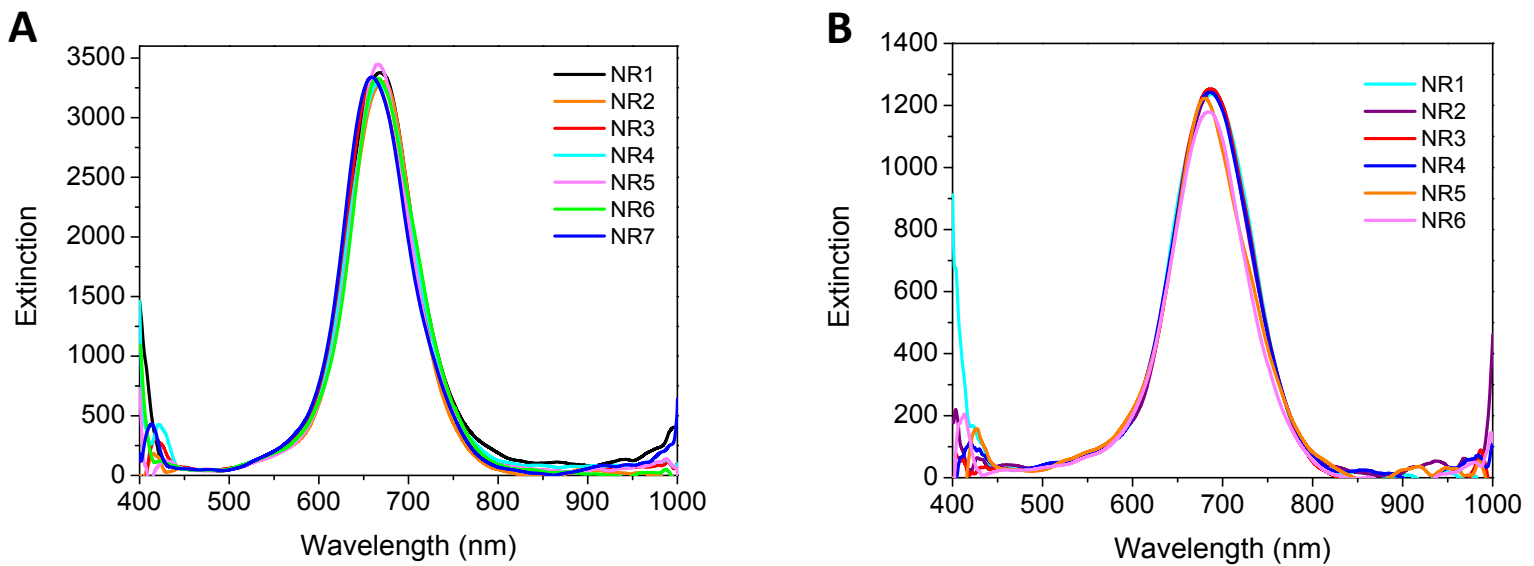

C
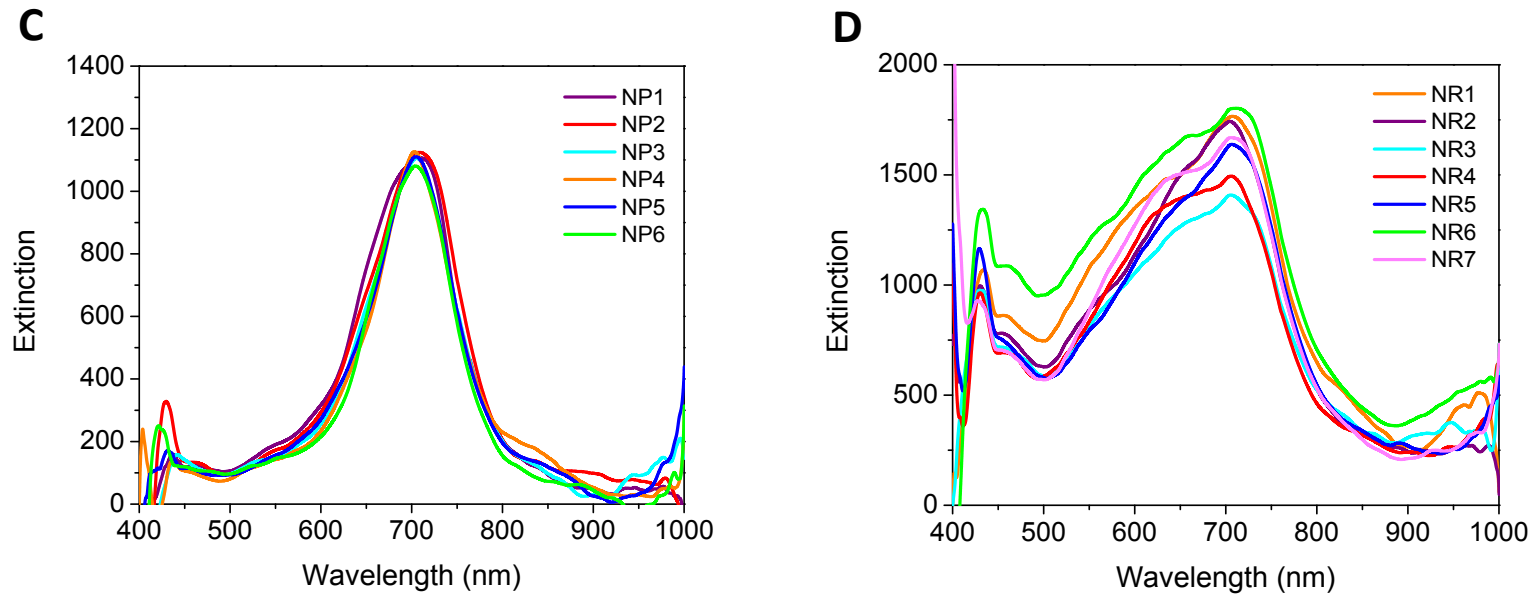

Figure S5. Single-particle extinction of several AuNRs and Pt@AuNRs: A) AuNRs, B) Pt@AuNRs-T, C) Pt@AuNRs-B, and D)Pt@AunRs-S. 

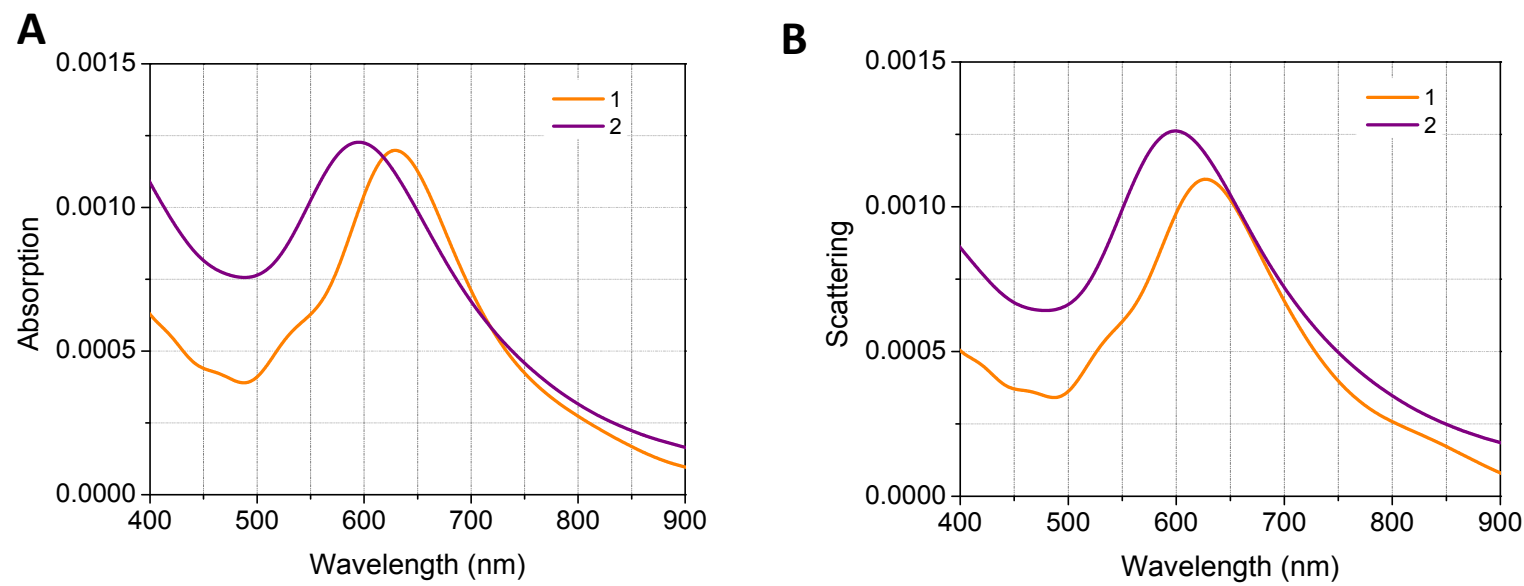

Figure S6. Calculated absorption (A) and scattering (B) of an Pt@AuNR covered by a continuous Pt layer with an increasing thickness. It is worth noting the generated peak at $\sim 545$ nm.
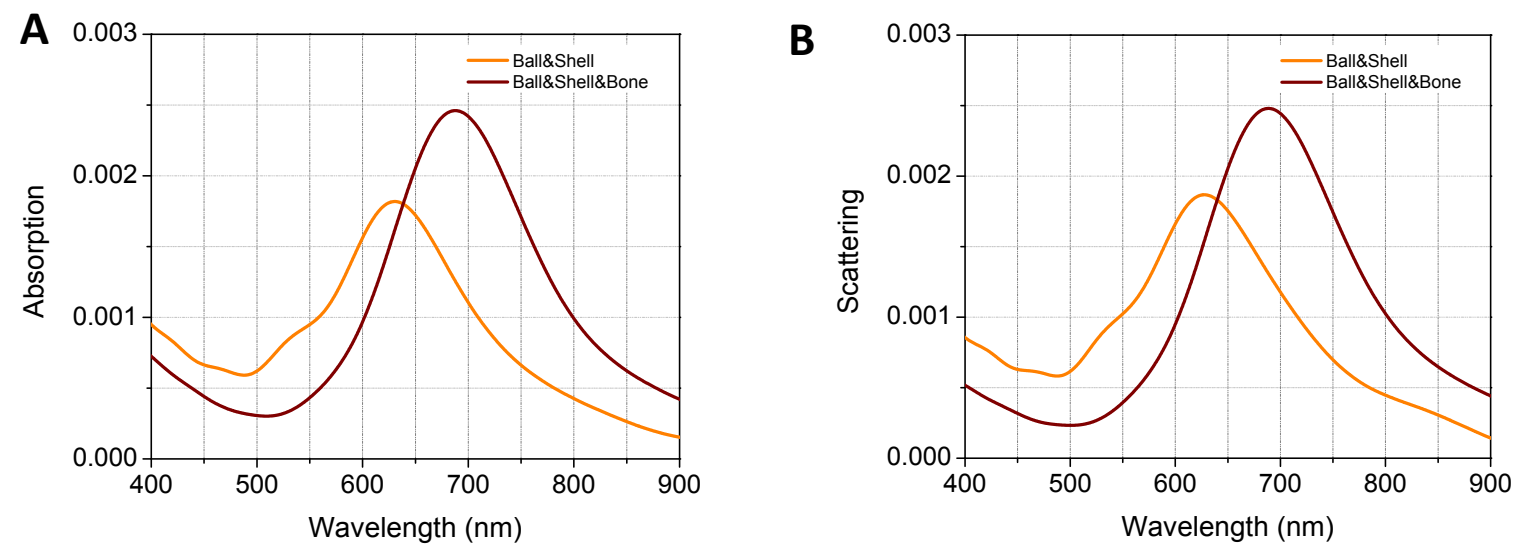

Figure S7. Calculated absorption (A) and scattering (B) of a Pt@AuNR-S covered by a Pt layer consisting of a thin shell and islands (orange); or a thin shell, islands and bone (purple). The peak at $\sim 545 \mathrm{~nm}$ is resembled. 


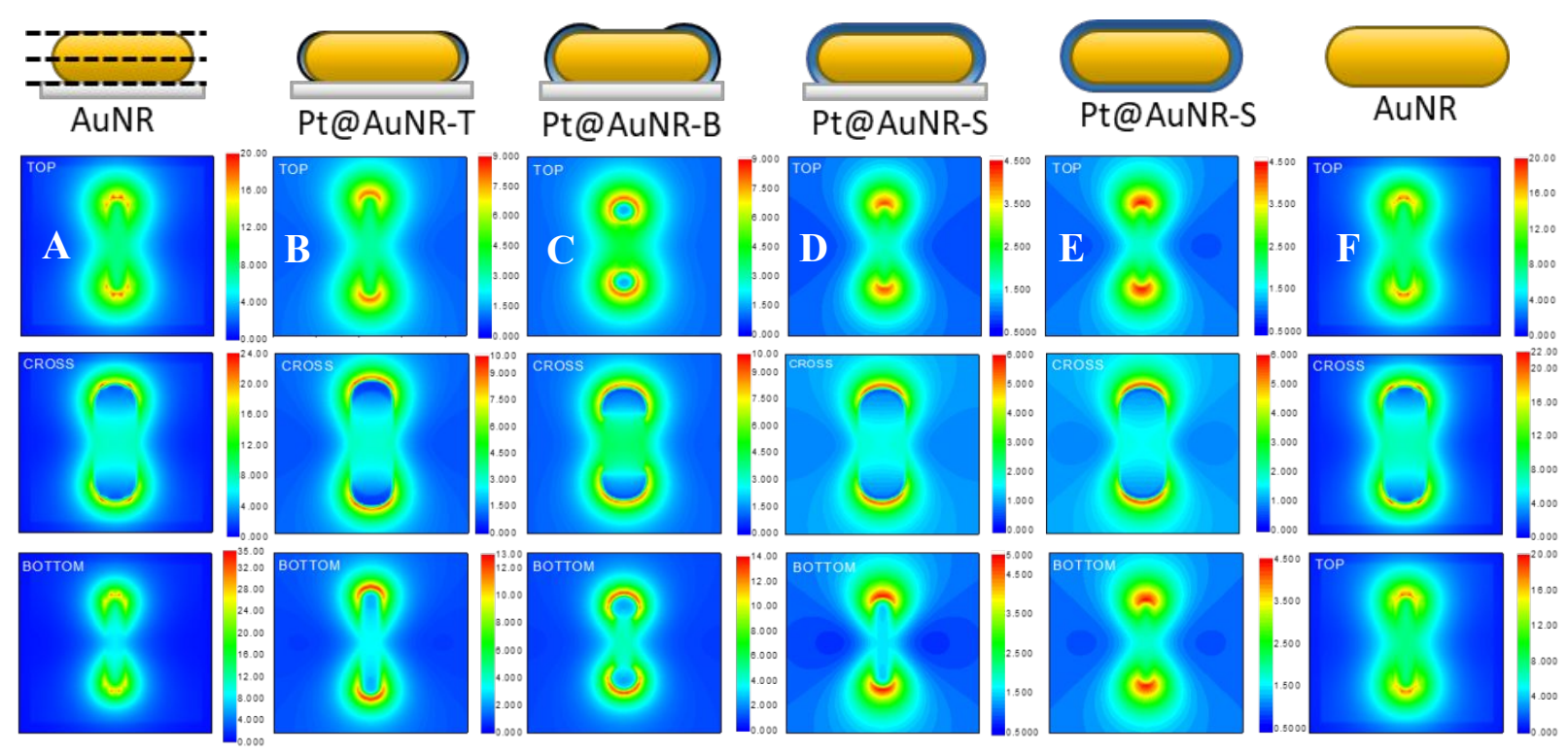

Figure S8. Electromagnetic plasmon field calculated on the top, middle, and bottom of: A) AuNR on a substrate, B) Pt@AuNR-T on a substrate, C) Pt@AuNR-B on a substrate, D) Pt@AuNR-S on a substrate, E) colloidal Pt@AuNR-S on water, and F) colloidal AuNR on water. 